\title{
Crest Factors of Complementary-Sequence-Based Multicode MC-CDMA Signals
}

\author{
Byoung-Jo Choi and Lajos Hanzo
}

\begin{abstract}
The crest factor properties of binary phase-shift keying modulated two- and four-code assisted multicarrier code-division multiple-access (MC-CDMA) employing complementary-sequence-based spreading sequences are characterized. More specifically, a complementary-sequence pair, a complementary-sequence-based subcomplementary code pair, and a Sivaswamy's complementary code set are studied. It was found that the corresponding crest factors are bounded by $3 \mathrm{~dB}$, which corresponds to the crest factor of a single sine wave. This low crest factor resulted in a lower power loss and a lower out-of-band power spectrum due to clipping when the time-domain signal was subjected to a typical nonlinear power amplifier, in comparison to those of Walsh code and orthogonal Gold code-spread MC-CDMA signals.
\end{abstract}

Index Terms-Complementary set, crest factor (CF), Golay codes, multicarrier code-division multiple-access (MC-CDMA), Shapiro-Rudin sequence, subcomplementary set.

\section{INTRODUCTION}

$\mathbf{T}$ HE fundamental problem of reducing the dynamic range of the sum of trigonometric series has been studied in numerous fields, such as in mathematics [1], [2], in radar engineering [3], [4], in measurement science [5], and in the context of orthogonal frequency-division multiplexing (OFDM) [6]. As a result, various binary and polyphase codes, such as Barker codes [7], Shapiro-Rudin sequences [1], Frank codes [8], Newman phases [2], Schroeder phases [9], and Zadoff-Chu codes [10], [11] have been investigated in order to arrive at a low crest factor.

The crest factors of multicarrier code-division multiple-access (MC-CDMA) [12], [13] signals have also been studied in [14] and [15], where each user is assigned a different subcarrier offset. The envelope power of the multicode MC-CDMA signal was also analyzed in [16] and it was shown that the combined aperiodic correlation properties of the spreading sequences employed characterize the envelope power, which can be viewed as a direct extension of Schroeder's early findings [9] in the context of a single-code scenario. A desirable set of spreading sequences designed for multicode MC-CDMA is expected to exhibit low values of aperiodic correlations, while exhibiting orthogonality between the sequences. While investigating the crest factor properties of multicode MC-CDMA employing various spreading sequences, it was found in [16] that the crest factors of multicode MC-CDMA employing Walsh codes can

Manuscript received November 17, 2001; revised May 20, 2002; accepted June 4, 2002. The editor coordinating the review of this paper and approving it for publication is C. Tellambura.

The authors are with the Department of Electronics and Computer Science, University of Southampton, Southampton SO17 1BJ, U.K. (e-mail: lh@ecs.soton.ac.uk).

Digital Object Identifier 10.1109/TWC.2003.819040 be bounded by $4 \mathrm{~dB}$ upon carefully selecting the code sets and upon employing high-rate crest-factor reduction coding, provided that the number of simultaneously used codes $L$ is higher than or equal to four. When $L$ is less than four, the MC-CDMA signals employing orthogonal Gold (OGold) codes, Frank codes [8], and Zadoff-Chu codes [10], [11] resulted in considerably lower crest factors. Even though Zadoff-Chu codes indeed do produce an ideal crest factor of less than $3 \mathrm{~dB}$ in the context of single-code MC-CDMA, the crest factor values of neither Zadoff-Chu codes, Frank codes, nor those of OGold codes are bounded by $3 \mathrm{~dB}$ in the context of two- and four-code-based MC-CDMA. It is widely recognized that complementary sequences, also known as Golay codes [17], produce crest factors bounded by $3 \mathrm{~dB}$ [18], [19], when they are applied to single-code MC-CDMA as in our context of interest [20], [21]. Ochiai and Imai [21] also reported that the employment of orthogonal complementary sequences in the context of multicode MC-CDMA resulted in low crest factors, which was shown using simulation studies. However, the crest factor properties of Shapiro-Rudin-based codes in the context of two- and four-code MC-CDMA signal have not been documented in the literature to the best of our knowledge.

In this letter, we characterize the advantageous crest factor (CF) properties of three complementary-sequence-based spreading sequences [17], namely those of a complementary-sequence pair [17], a complementary-sequence-based subcomplementary pair [3], and those of a Sivaswamy's complementary set [3], in the context of both two- and four-code binary phase-shift keying (BPSK) modulated MC-CDMA schemes in order for transmitting two and four bits simultaneously. The scenario investigated can also be encountered in the context of multipulse radar signals [4].

The letter is organized as follows. Section II introduces the multicode MC-CDMA system model considered. Section III presents the crest factor properties of three complementary-sequence-based spreading sequences. The effects of clipping in terms of the output power reduction and the out-of-band spurious power of multicode MC-CDMA signals employing complementary-sequence-based spreading sequences are presented in Section IV in comparison to those of other spreading sequences, followed by our conclusions in Section V.

\section{SySTEM MOdEL AND ENVELOPE POWER}

The simplified transmitter structure of the multicode MC-CDMA system considered is portrayed in Fig. 1. Each of the $L$ number of input bits $b_{0}, b_{1}, \ldots, b_{L-1}$ is spread with the aid of an $N$-chip spreading code selected from the set $\left\{\mathbf{C}_{0}, \mathbf{C}_{1}, \ldots, \mathbf{C}_{L-1}\right\}$. Then the resultant $L$ number of modulated spreading codes are superimposed and each of 


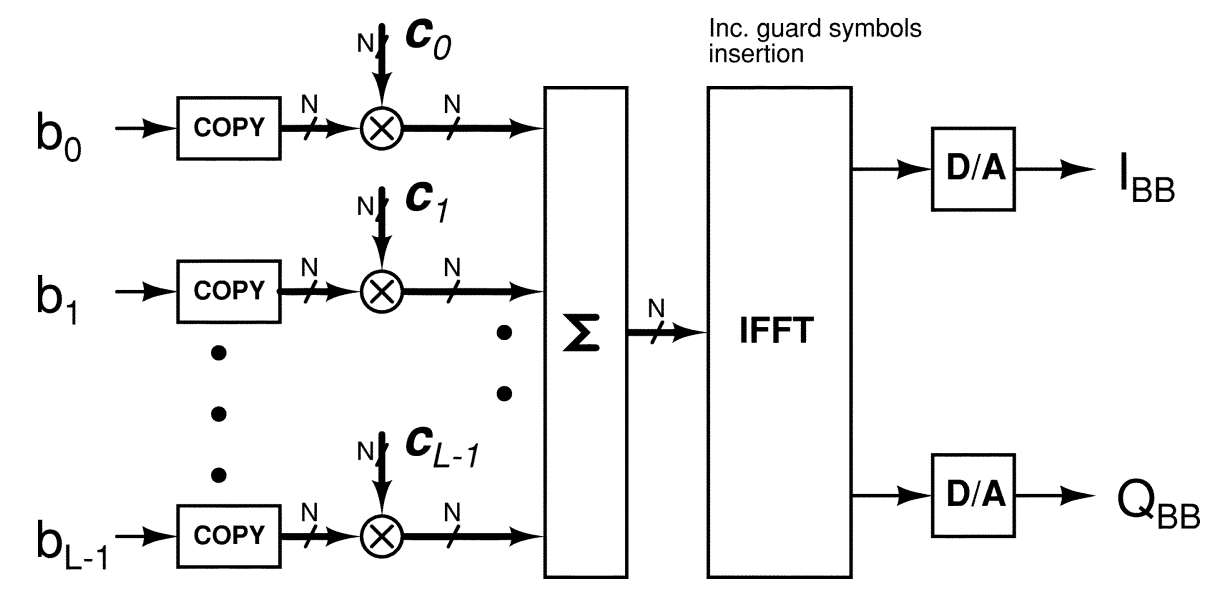

Fig. 1. Multicode MC-CDMA transmitter schematic.

the composite chips is mapped to one of the $N$ subcarriers. Finally, $N$-point inverse fast Fourier transform (IFFT)-based modulation is used for generating the modulated in-phase $(I)$ and quadrature-phase $(Q)$ signals of Fig. 1. The system model described above may correspond to the downlink scenario of an MC-CDMA base station or to the uplink scenario of mobiles transmitting multiple bits combined with time-division multiple-access or orthogonal frequency-division multiple-access. Although the attractive family of quadrature amplitude modulation schemes [22] can be readily used for generating the information symbols $\left\{b_{l}\right\}$ from the source data bits, here we limit the symbol mapping scheme to BPSK. Furthermore, we limit our choice of the spreading sequences to those having elements satisfying $\left|c_{l}[n]\right|=1$. The normalized complex envelope of a BPSK modulated multicode MC-CDMA signal may be represented for the duration of a symbol period $T$ as

$$
s^{(L)}(t)=\frac{1}{\sqrt{N}} \sum_{l=0}^{L-1} \sum_{n=0}^{N-1} b_{l} c_{l}[n] e^{j 2 \pi F n(t / T)}
$$

where $L$ is the number of simultaneously transmitted symbols and $F$ is the subcarrier separation parameter [12] invoked for mapping the $N$-chip spread information symbol to $N$ subcarriers that are sufficiently far apart in the frequency domain, in order to experience independent fading of the different subcarriers.

The CF of the multicode MC-CDMA signal $s(t)$ is defined as the ratio of the peak to the root mean square amplitude [18]

$$
\mathrm{CF} \triangleq \frac{\|s\|_{\infty}}{\|s\|_{2}}
$$

where $\|s\|_{\infty}=\max _{t}|s(t)|$ and $\|s\|_{2}=\sqrt{1 / T \int_{T}|s(t)|^{2} d t}=$ $L$. The $\mathrm{CF}$ values are often represented in terms of decibels as $20 \log _{10}(\mathrm{CF})$. Sometimes the peak factor (PF) or peak-to-mean envelope power ratio (PMEPR) is used instead of the $\mathrm{CF}$, which are defined as $\mathrm{PF}=\mathrm{PMEPR} \triangleq(\mathrm{CF})^{2}$.

\section{COMPLEMENTARY SEQUenCE-BASED SPREADING SEQUENCES}

Let us denote the $z$-transform of a sequence $\mathbf{s}=$ $\left(s_{n}[0] s_{n}[1] s_{n}[2] \ldots s_{n}[N-1]\right)$ as $\mathbf{S}(z)$, which is defined as $\mathbf{S}(z) \triangleq \sum_{n=0}^{N-1} s[n] z^{n}$. Then, we can readily verify that $\mathbf{S}^{*}(z) \mathbf{C}(z)=\sum_{n=-N+1}^{N-1} R_{\mathrm{s}, \mathrm{c}}[n]$, where $R_{\mathrm{s}, \mathrm{c}}[n]$ is the aperiodic crosscorrelation between the sequences $\mathbf{S}$ and $\mathbf{c}$, defined as $R_{\mathbf{s}, \mathbf{c}}[n] \triangleq \sum_{i=0}^{N-n-1} s^{*}[i] c[i+n]$. When the two sequences are identical, i.e., we have $\mathbf{s}=\mathbf{c}$, the $z$-transform of the corresponding autocorrelation function $R_{\mathrm{S}}[n] \triangleq \sum_{i=0}^{N-n-1} s^{*}[i] s[i+n]$ can be represented as $\left|\mathbf{S}^{*}(z)\right|^{2}$. Then, a pair of equally long sequences $\{\mathbf{s}, \mathbf{c}\}$ is said to be a complementary pair, if and only if [17], [19]

$$
|\mathbf{S}(z)|^{2}+|\mathbf{C}(z)|^{2}=2 N
$$

on the boundary of the unit disc in the complex plane, i.e., at $|z|=1$.

\section{A. Two-Code Case}

Let $\mathbf{c}_{0}$ and $\mathbf{c}_{1}$ be two spreading sequences of length $N$, employed for transmitting the information bits $b_{0}$ and $b_{1}$, respectively. Then, the normalized complex envelope of the BPSK modulated two-code MC-CDMA signal $s^{(2)}(t)$ of (1) can be expressed as

$$
\begin{aligned}
s^{(2)}(t) & =\frac{1}{\sqrt{N}}\left\{b_{0} \sum_{n=0}^{N-1} c_{0}[n] e^{j 2 \pi F n(t / T)}\right. \\
& \left.+b_{1} \sum_{n=0}^{N-1} c_{1}[n] e^{j 2 \pi F n(t / T)}\right\} \\
= & \frac{1}{\sqrt{N}}\left\{b_{0} \mathbf{C}_{0}(z)+b_{1} \mathbf{C}_{1}(z)\right\}
\end{aligned}
$$

where the superscript in $s^{(2)}(t)$ was used for denoting the number of simultaneously transmitted codes $L=2$ and we used $z=e^{j 2 \pi F n(t / T)}$.

Lemma 1: The crest factor of the two-code BPSK MC-CDMA signal employing a complementary pair of sequences as its spreading sequence is bounded by $3 \mathrm{~dB}$.

Proof: Let $\mathbf{s}$ and $\mathbf{c}$ be the pair of complementary sequences of length $N$. Then, the envelope power of $s^{(2)}(t)$ given in (5) can be expressed as

$$
\begin{aligned}
\left|s^{(2)}(t)\right|^{2} & =\frac{1}{N}\left|b_{0} \mathbf{S}(z)+b_{1} \mathbf{C}(z)\right|^{2} \\
& \leq \frac{2}{N}\left\{|\mathbf{S}(z)|^{2}+|\mathbf{C}(z)|^{2}\right\} \\
& =4
\end{aligned}
$$


where we used the property of a pair of complementary sequences given in (3) and the inequality relationship of $b_{0} \mathbf{S}^{*}(z) b_{1} \mathbf{C}(z)+b_{0} \mathbf{S}(z) b_{1} \mathbf{C}^{*}(z) \leq|\mathbf{S}(z)|^{2}+|\mathbf{C}(z)|^{2}$. Since the average power of a two-code BPSK modulated MC-CDMA signal is $L=2$, the corresponding PF is bounded by two. I

We would like to point out that although Lemma 1 was presented in the context of BPSK modulation for the sake of consistency with other lemmas, it can be readily shown that Lemma 1 holds also for the more generic class of $M$-ary phase shift keying modulated signals. Furthermore, it can be shown $^{1}$ that when $\mathbf{s}$ and $\mathbf{c}$ are two arbitrary complementary sequences having a length of $N$, which are not necessarily a complementary pair, the corresponding crest factor is bounded by $6 \mathrm{~dB}$ in the same scenario as that in Lemma 1 .

Sivaswamy introduced subcomplementary sequences in the context of radar signal design [3]. A pair of sequences of length $N=2^{k} N_{o}, k \geq 1$, is referred to as a subcomplementary pair, if the sum of their aperiodic autocorrelations is zero for $n>$ $N_{o}$. A method of constructing subcomplementary pairs was also given in [3] as follows. Let $\mathbf{c}$ be an arbitrary sequence of length $N / 2$. Then, a subcomplementary pair of sequences $\mathbf{s}$ and $\hat{\mathbf{s}}$ is constructed as [3]

$$
\mathrm{s}=\mathbf{c c} \quad \hat{\mathrm{s}}=\mathbf{c} \overline{\mathbf{c}}
$$

where $\mathbf{c c}$ denotes a concatenated sequence of the two identical sequences $\mathbf{c}$, while $\overline{\mathbf{c}}$ represents a negated version of the original sequence c. Sivaswamy's subcomplementary pairs exhibit similar crest factor characteristics to those of the complementary-sequence pair in the context of two-code BPSK modulated MC-CDMA systems.

Lemma 2: If we apply a complementary sequence [17] of length $N / 2$ as the base sequence $\mathbf{c}$, then the subcomplementary pair [3] $\mathbf{s}$ and $\hat{\mathbf{s}}$ of (8) produces a crest factor of less than or equal to $3 \mathrm{~dB}$, provided that they are applied in two-code BPSK modulated MC-CDMA systems.

Proof: The $z$-transforms of $\mathbf{s}$ and $\hat{\mathbf{s}}$ can be expressed as

$$
\begin{aligned}
& \mathbf{S}(z)=\mathbf{C}(z)+z^{N / 2} \mathbf{C}(z) \\
& \hat{\mathbf{S}}(z)=\mathbf{C}(z)-z^{N / 2} \mathbf{C}(z)
\end{aligned}
$$

where $\mathbf{C}(z)$ is the $z$-transform of a complementary sequence of length $N / 2$. Hence, $s^{(2)}(t)$ of (5) is given as

$$
\begin{aligned}
s^{(2)}(t) & =\frac{1}{\sqrt{N}}\left\{\left(b_{0}+b_{1}\right) \mathbf{C}(z)+\left(b_{0}-b_{1}\right) z^{N / 2} \mathbf{C}(z)\right\} \\
& = \begin{cases}\frac{2}{\sqrt{N}} b_{0} \mathbf{C}(z), & \text { when } b_{0}=b_{1} \\
\frac{2}{\sqrt{N}} b_{0} z^{N / 2} \mathbf{C}(z), & \text { otherwise. }\end{cases}
\end{aligned}
$$

The envelope power $\left|s^{(2)}(t)\right|^{2}$, evaluated at $|z|=1$, is given as

$$
\left|s^{(2)}(t)\right|^{2}=\frac{4}{N}|\mathbf{C}(z)|^{2} \leq 4
$$

where we exploited the fact that $|\mathbf{C}(z)|^{2} \leq N$ on $|z|=1$ [19]. Hence, the corresponding PF is bounded by two.

${ }^{1}$ The authors would like to thank Prof. C. Tellambura for pointing out this extension.

\section{B. Four-Code Case}

So far we have concentrated our attention on the specific cases, when two symbols are transmitted simultaneously. Let us now extend our discussions to the scenario where four spreading sequences are used simultaneously. Let $\left\{\mathbf{c}_{1}, \mathbf{c}_{2}, \mathbf{c}_{3}, \mathbf{c}_{4},\right\}$ be a set of four spreading sequences of length $N$, employed for transmitting the information bits $b_{1}, b_{2}, b_{3}$, and $b_{4}$, respectively. Then, the normalized complex envelope of the BPSK modulated four-code MC-CDMA signal $s^{(4)}(t)$ of (1) can be expressed as

$$
s^{(4)}(t)=\frac{1}{\sqrt{N}}\left\{b_{1} \mathbf{C}_{1}(z)+b_{2} \mathbf{C}_{2}(z)+b_{3} \mathbf{C}_{3}(z)+b_{4} \mathbf{C}_{4}(z)\right\}
$$

where, again, the superscript ${ }^{(4)}$ denotes the number of simultaneously transmitted codes $L=4$ and we used $z=e^{j 2 \pi F n(t / T)}$.

Tseng and Liu [23] introduced a wider range of complementary sets of sequences. A set of sequences of equal length is said to be a complementary set, if the sum of autocorrelations of all the sequences in the set is zero, except for the peak term at the zero-shift position [23]. Sivaswamy [3] proposed a method for constructing a complementary set of $2^{n+1}$ number of sequences of length $2^{n} N$, where $n \geq 1$ and $N$ is the length of the base complementary pair. When $n=1$, we can obtain a complementary set of four sequences of length $N$ from a base complementary pair $\mathbf{s}_{0}$ and $\mathbf{c}_{0}$ of length $N / 2$ using the following method [3]:

$$
\mathbf{c}_{1}=\mathbf{s}_{0} \mathbf{s}_{0} \quad \mathbf{c}_{2}=\mathbf{s}_{0} \overline{\mathbf{s}}_{0} \quad \mathbf{c}_{3}=\mathbf{c}_{0} \mathbf{c}_{0} \quad \mathbf{c}_{4}=\mathbf{c}_{0} \overline{\mathbf{c}}_{0} .
$$

Lemma 3: If we apply a pair of complementary sequences [17] of length $N / 2$ as the base sequences $\mathbf{s}_{0}$ and $\mathbf{c}_{0}$, the set of four complementary sequences, given by (15), produces a crest factor of less than or equal to $3 \mathrm{~dB}$, provided that the sequences $\mathbf{c}_{1}, \mathbf{c}_{2}, \mathbf{c}_{3}$, and $\mathbf{c}_{4}$ are applied to four-code BPSK modulated MC-CDMA systems.

Proof: The corresponding $z$-transforms of the spreading sequences employed can be expressed as

$$
\begin{aligned}
& \mathbf{C}_{1}(z)=\mathbf{S}_{0}(z)+z^{N / 2} \mathbf{S}_{0}(z) \\
& \mathbf{C}_{2}(z)=\mathbf{S}_{0}(z)-z^{N / 2} \mathbf{S}_{0}(z) \\
& \mathbf{C}_{3}(z)=\mathbf{C}_{0}(z)+z^{N / 2} \mathbf{C}_{0}(z) \\
& \mathbf{C}_{4}(z)=\mathbf{C}_{0}(z)-z^{N / 2} \mathbf{C}_{0}(z) .
\end{aligned}
$$

Upon substituting (16) - (19) into (14), we have four possible expressions of $\sqrt{N} s^{(4)}(t)$ depending on the four information bits, which are given as

$$
\begin{aligned}
& \sqrt{N} s^{(4)}(t) \\
& = \begin{cases}2\left(b_{1} \mathbf{S}_{0}(z)+b_{3} \mathbf{C}_{0}(z)\right), & \text { when } b_{1}=b_{2} \text { and } b_{3}=b_{4} \\
2 z^{N / 2}\left(b_{1} \mathbf{S}_{0}(z)+b_{3} \mathbf{C}_{0}(z)\right), & \text { when } b_{1} \neq b_{2} \text { and } b_{3} \neq b_{4} \\
2\left(b_{1} \mathbf{S}_{0}(z)+b_{3} z^{N / 2} \mathbf{C}_{0}(z)\right), & \text { when } b_{1}=b_{2} \text { and } b_{3} \neq b_{4} \\
2\left(b_{1} z^{N / 2} \mathbf{S}_{0}(z)+b_{3} \mathbf{C}_{0}(z)\right), & \text { when } b_{1} \neq b_{2} \text { and } b_{3}=b_{4} .\end{cases}
\end{aligned}
$$

Considering that the length of the sequences $\mathbf{s}_{0}$ and $\mathbf{c}_{0}$ is $N / 2$, it can be readily shown that the corresponding envelope power satisfies

$$
N\left|s^{(4)}(t)\right|^{2}=8\left(\left|\mathbf{S}_{0}(z)\right|^{2}+\left|\mathbf{C}(z)_{0}\right|\right) \leq 8 N
$$

for all the above four cases, using similar techniques to those employed in the proofs of Lemmas 1 and 2. Hence, we have 


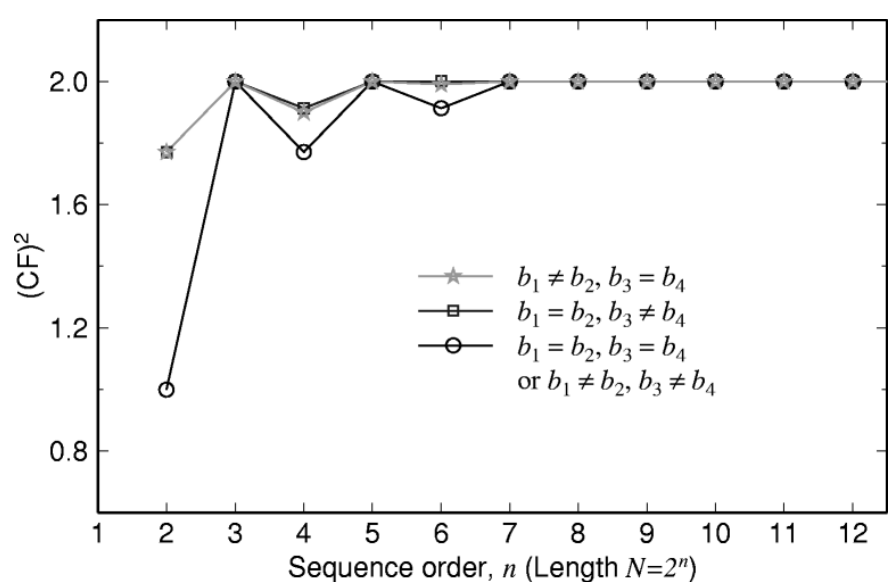

Fig. 2. Crest-factors of Sivaswamy's complementary set of sequences. The number of simultaneously used sequences is $L=4$ and the sequence length of $N=2^{n}$ is between $2^{2}=4$ and $=2^{13}=8192$.

$\left|s^{(4)}(t)\right|^{2} \leq 8$. Since the average power of $s^{(4)}(t)$ is $L=4$, the $\mathrm{PF}$ is bounded by two.

Fig. 2 depicts the PFs of some of Sivaswamy's complementary sets constituted by $L=4$ sequences, where the oversampling factor of eight was employed to calculate accurate $\mathrm{PF}$ values [24]. Depending on the relationship between the message sequences depicted in (20), the corresponding crest factors exhibit three different values, as seen in Fig. 2. Half the number of the message sequences belong to the case represented by the marker "o" and a quarter to those denoted by each of the marker " $\square$ " and " $\star$." We can observe in Fig. 2 that the PFs associated with the marker "o" are the lowest among the three values, when the sequence length $N$ is $2^{2}=4,2^{4}=16$, or $2^{6}=64$. An ideal PF of one was achieved for the sequence length of $N=2^{2}=4$, representing a constant power envelope. The fact that all the crest factors in Fig. 2 are bounded by $3 \mathrm{~dB}$ corroborates Lemma 3.

It remains, however, an open question, whether there exists a large set of complementary spreading sequences, where the number of codes $L$ is higher than four, exhibiting low crest factors. For example, we can construct a set of eight orthogonal subcomplementary sequences from the Kronecker product of base complementary sequences and a $4 \times 4$ Walsh matrix [4]. However, the corresponding maximum PF of BPSK modulated eight-code MC-CDMA was 3.49, which is considerably higher than that of the best-case Walsh code spread MC-CDMA scheme, as seen in Fig. 3. In fact, Walsh spreading codes represented by the $16 \times 16$ Walsh-Hadamard matrix constitute an orthogonal complementary set [4]. As the number of codes $L$ increases in multicode MC-CDMA, the interaction of the message bits with the pair-wise crosscorrelation property of the sequences becomes complex [16]. Hence, it becomes an arduous task to arrive at a predictable behavior in terms of the corresponding envelope power.

\section{EFFECTS OF CLIPPING}

In the previous section, we observed that two- and four-code BPSK modulated MC-CDMA signals employing complementary-sequence-based spreading sequences result in low values of the corresponding crest factor, which are bounded by $3 \mathrm{~dB}$. Some of the practical consequences of this low crest factor will

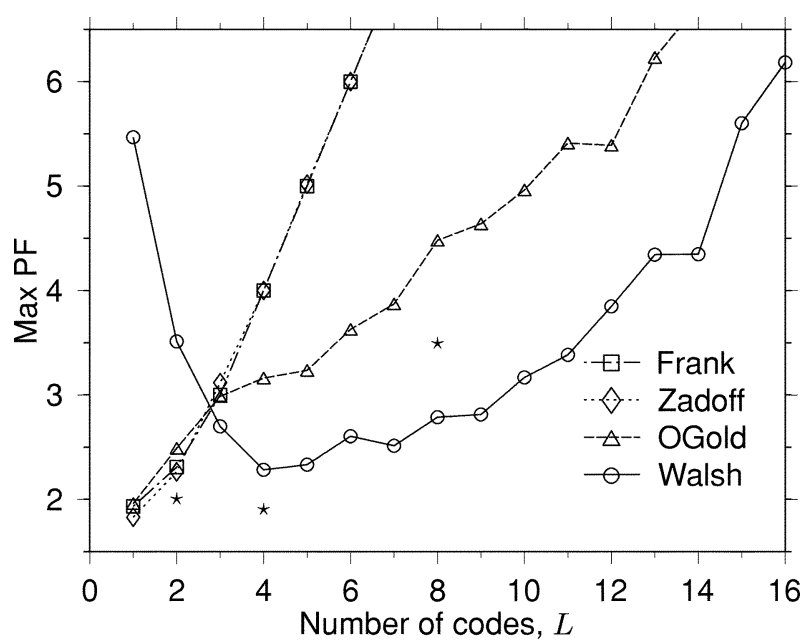

Fig. 3. Maximum $\mathrm{PF}\left(\mathrm{CF}^{2}\right)$ when using various spreading codes for BPSK modulated multicode MC-CDMA. The spreading factor was $N=16$. The markers " $\star$ " represent the corresponding maximum PFs of complementary-sequence-based spreading sequences investigated.

be investigated here in comparison to other sequences used for spreading MC-CDMA signals. When they are subjected to nonlinear amplification, they produce spurious out-of-band emissions. The extreme peaks of the signal inevitably suffer from so-called "clipping effects," when they enter the saturation region of the amplifier, resulting in the loss of effective transmission power as well as in signal distortion unless the gain of the amplifier is reduced to a level, where the amplifier's maximum output level is sufficiently high for the signal peaks not to be clipped. Many portable wireless devices use solid-state power amplifiers (SSPA). The amplitude transfer characteristics or the amplitude modulation-amplitude modulation curve $g(x)$ of an SSPA can be modeled as [25], [26]

$$
g(x)=\frac{x}{\left(1+x^{2 p}\right)^{1 / 2 p}}
$$

where $x$ is the input magnitude and $p$ is the smoothness factor during its transition from the linear region to the saturation region. Considering that the output power of typical SSPAs at the 1-dB compression point is about $0.7 \mathrm{~dB}$ below the saturation power, the value of the smoothness factor in (22) becomes $p=3.58$.

The power loss due to the above-mentioned "clipping effects" of the nonlinear amplifier model of (22) can be observed in Figs. 4 and 5. For the two-code BPSK modulated MC-CDMA schemes characterized in Fig. 4(a), the MC-CDMA signal employing a complementary pair of sequences showed the lowest power loss of $1.6 \mathrm{~dB}$ for the set of five different spreading codes investigated. A pair of OGold codes and a pair of Frank codes also exhibited a similar behavior, showing an associated power loss of 1.7 and $1.8 \mathrm{~dB}$, respectively. Finally, the Walsh codes and Zadoff-Chu codes exhibited a high power loss of around $3 \mathrm{~dB}$ at 0 -dB input backoff. The spreading code pairs were selected on the basis of maintaining the lowest crest-factors for each family of spreading codes.

When four spreading codes were used simultaneously, as seen in Fig. 4(b), the effective transmission power losses at 0-dB input backoff were $1.3 \mathrm{~dB}$ for Sivaswamy's complementary set, 


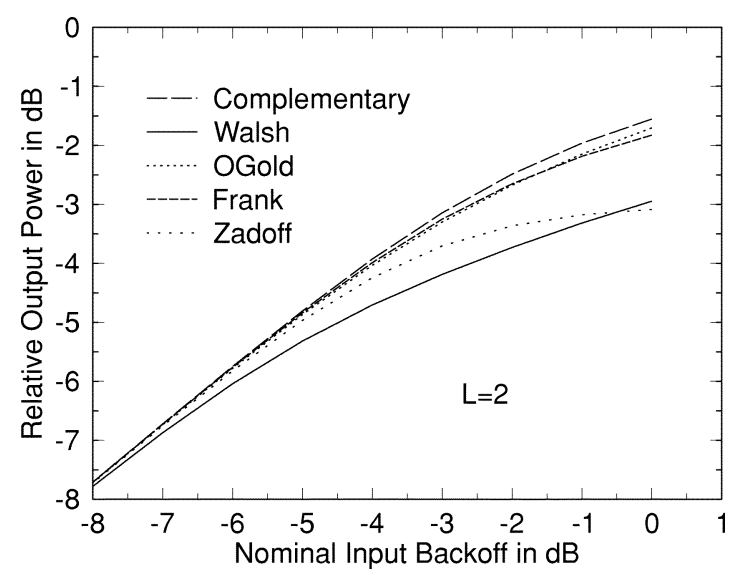

(a)

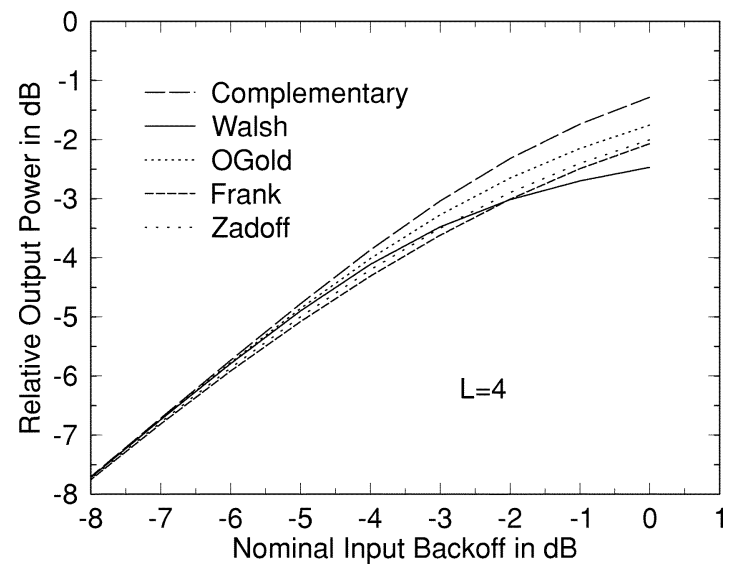

(b)

Fig. 4. Relative output power versus input backoff in multicode BPSK modulated MC-CDMA employing various spreading codes of length $N=16$.

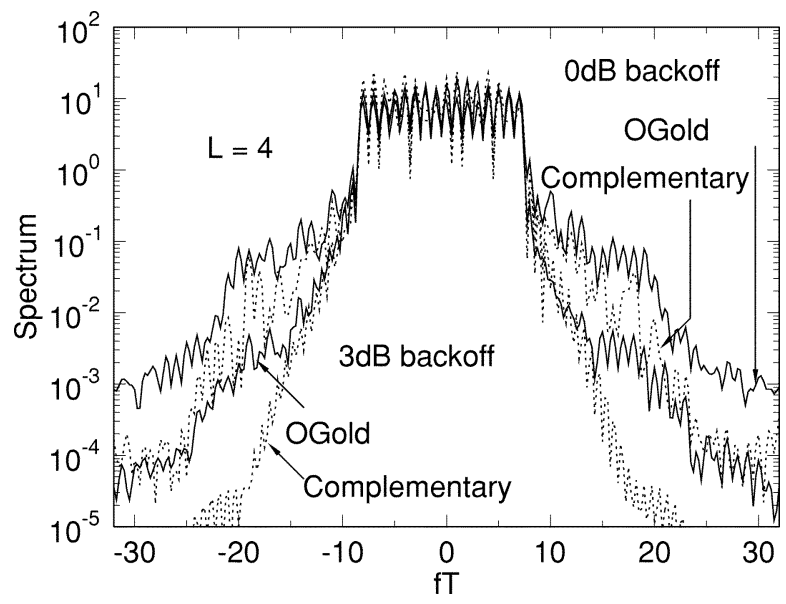

Fig. 5. Relative output power versus input backoff in four-code BPSK modulated MC-CDMA employing OGold codes and Sivaswamy's complementary set of sequences having the length of $N=16$.

$1.8 \mathrm{~dB}$ for OGold codes, $2.0 \mathrm{~dB}$ for Zadoff-Chu codes, $2.1 \mathrm{~dB}$ for Frank codes, and $2.5 \mathrm{~dB}$ for Walsh codes. Considering that the best combination of Walsh codes exhibited a lower maximum CF than that of the OGold codes, as seen in Fig. 3, the Walsh-code-related high power loss is surprising. This implies that the Walsh-spread four-code MC-CDMA signal still exhibits a relatively poor magnitude distribution.
The nonlinear amplification of MC-CDMA signals generates high out-of-band power spectrum. Fig. 5 compares the power spectra of four-code BPSK modulated MC-CDMA signals employing OGold codes and Sivaswamy's complementary sets of length $N=16$. Raised cosine shaping was used at the boundaries of a transmission frame for reducing the out-of-band power. All the message sequences were applied and their corresponding spectra were averaged for generating Fig. 5. The spectrum corresponding to Sivaswamy's complementary set exhibits the lowest out-of-band power spillage. The spectrum of the Walsh-spread scheme fell between that of the OGold codes and that of Sivaswamy's complementary sets.

\section{CONCLUSION}

The advantageous crest factor properties of complementary sequences recorded in the context of single-code MC-CDMA as well as OFDM have been widely recognized. In this letter, the crest factor values of two- and four-code BPSK modulated MC-CDMA signals employing three different complementary-sequence-based spreading sequences, namely a complementary pair, a complementary code-based subcomplementary pair and one of Sivaswamy's complementary sets, were shown to be bounded by $3 \mathrm{~dB}$, regardless of the sequence length. It was demonstrated that the corresponding low crest factor of the MC-CDMA signal employing complementary-sequence-based spreading sequences, in comparison to Walsh- and OGold-spread MC-CDMA signals, is practically important, since signals exhibiting a low crest factor result in lower power loss and lower out-of-band emissions, when the signal is subjected to nonlinear amplification. However, the investigated scenario is limited to two- and four-code-based MC-CDMA signals employing BPSK modulation, and hence, further research efforts are required for finding a sufficiently large set of spreading sequences having desirable crest factor properties.

\section{ACKNOWLEDGMENT}

The authors would like to thank the anonymous reviewers for their valuable suggestions. Furthermore, the authors are particularly grateful to Prof. C. Tellambura for suggesting elegant and simple proofs of Lemmas 1-3, which substantially improved the presentation of the letter.

\section{REFERENCES}

[1] W. Rudin, "Some theorems on Fourier coefficients," in Proc. Ame. Mathematics Society, vol. 10, Dec. 1959, pp. 855-859.

[2] D. J. Newman, "An $L^{1}$ extrimal problem for polynomials," in Proc. Ame. Mathematics Society, vol. 16, Dec. 1965, pp. 1287-1290.

[3] R. Sivaswamy, "Digital and analog subcomplementary sequences for pulse compression," IEEE Trans. Aerosp. Electron. Syst., vol. AES-14, pp. 343-350, Mar. 1978.

[4] F. F. Kretschmer, Jr. and K. Gerlach, "Low sidelobe radar waveforms derived from orthogonal matrices," IEEE Trans. Aerosp. Electron. Syst., vol. 27, pp. 92-101, Jan. 1991.

[5] E. Van der Ouderaa, J. Schoukens, and J. Renneboog, "Peak factor minimization using a time-frequency domain swapping algorithm," IEEE Trans. Instrum. Meas., vol. 37, pp. 145-147, Mar. 1988.

[6] A. E. Jones, T. A. Wilkinson, and S. K. Barton, "Block coding scheme for reduction of peak to mean envelope power ratio of multicarrier transmission schemes," Electron. Lett., vol. 30, no. 25, pp. 2098-2099, Dec. 1994. 
[7] R. H. Barker, "Group synchronizing of binary digital systems," Commun. Theory, pp. 273-287, 1953.

[8] R. L. Frank, "Polyphase codes with good nonperiodic correlation properties," IEEE Trans. Inform. Theory, vol. IT-9, pp. 43-45, Jan. 1963

[9] M. R. Schroeder, "Synthesis of low-peak-factor signals and binary sequences with low autocorrelation," IEEE Trans. Inform. Theory, vol. IT-16, pp. 85-89, Jan. 1970.

[10] D. C. Chu, "Polyphase codes with good periodic correlation properties," IEEE Trans. Inform. Theory, vol. IT-18, pp. 531-532, July 1972.

[11] R. L. Frank, "Comments on polyphase codes with good correlation properties," IEEE Trans. Inform. Theory, vol. IT-19, p. 244, Mar. 1973.

[12] N. Yee, J. P. Linnartz, and G. Fettweis, "Multicarrier CDMA in indoor wireless radio networks," in Proc. PIMRC, 1993, pp. 109-113.

[13] K. Fazel and L. Papke, "On the performance of convolutionally-coded CDMA/OFDM for mobile communication system," in Proc. PIMRC, 1993, pp. 468-472.

[14] V. Aue and G. P. Fettweis, "Multicarrier spread spectrum modulation with reduced dynamic range," in Proc. VTC, Apr./May 1996, pp. 914-917.

[15] B. M. Popović, "Spreading sequences for multicarrier CDMA systems," IEEE Trans. Commun., vol. 47, pp. 918-926, June 1999.

[16] B. J. Choi, E. L. Kuan, and L. Hanzo, "Crest-factor study of MC-CDMA and OFDM," in Proc. VTC (Fall), 1999, pp. 233-237.

[17] M. J. E. Golay, "Complementary series," IRE Trans. Inform. Theory, vol. IT-7, pp. 82-87, Apr. 1961.
[18] S. Boyd, "Multitone signals with low crest factor," IEEE Trans. Circuits Syst., vol. CAS-33, pp. 1018-1022, Oct. 1986.

[19] B. M. Popović, "Synthesis of power efficient multitone signals with flat amplitude spectrum," IEEE Trans. Commun., vol. 39, pp. 1031-1033, July 1991.

[20] T. F. Ho and V. K. Wei, "Synthesis of low-crest waveforms for multicarrier CDMA system," in Proc. GLOBECOM, 1995, pp. 131-135.

[21] H. Ochiai and H. Imai, "OFDM-CDMA with peak power reduction based on the spreading sequences," in Proc. ICC, 1998, pp. 1299-1303.

[22] L. Hanzo, W. T. Webb, and T. Keller, Single- and Multi-carrier Quadrature Amplitude Modulation. New York: IEEE Press-Wiley, 2000.

[23] C.-C. Tseng and C. L. Liu, "Complementary sets of sequences," IEEE Trans. Inform. Theory, vol. IT-18, pp. 644-652, Sept. 1972.

[24] C. Tellambura, "Computation of the continuous-time PAR of an OFDM signal with BPSK subcarriers," IEEE Commun. Lett., vol. 5, pp. 185-187, May 2001.

[25] E. Costa, M. Midrio, and S. Pupolin, "Impact of amplifier nonlinearities on OFDM transmission system performance," IEEE Commun. Lett., vol. 3, pp. 37-39, Feb. 1999.

[26] G. Santella and F. Mazzanga, "A model for performance evaluation in M-QAM-OFDM schemes in presence of nonlinear distortions," in Proc. VTC, July 1995, pp. 830-834. 